\title{
A Shannon Entropy Approach for Structural Damage Identification based on Self-powered Sensor Data
}

\author{
Mohsen Mousavi ${ }^{\mathrm{a}}$, Damien Holloway ${ }^{\mathrm{a}}$, J.C. Oliviera ${ }^{\mathrm{a}}$, Amir H. Alavi ${ }^{\mathrm{b}}$, Amir \\ H. Gandomi *c,d \\ ${ }^{a}$ College of Sciences and Engineering, University of Tasmania, Hobart 7005, Tasmania, \\ Australia \\ ${ }^{b}$ Department of Civil and Environmental Engineering, University of Missouri, Columbia, \\ MO 65211, USA \\ ${ }^{c}$ Faculty of Engineering and IT, University of Technology Sydney, Ultimo, NSW 2007, \\ Australia \\ ${ }^{d}$ Stevens Institute of Technology, Hoboken, NJ 07030, USA
}

\begin{abstract}
Piezo-floating-gate (PFG) sensors are a class of self-powered sensors fabricated using piezoelectric transducers and p-channel floating-gate metaloxide-semiconductor (pMOS) transistors. These sensors are equipped with a series of floating-gates that are triggered when the voltage generated by the piezoelectric transducers exceeds one of the specified thresholds. Upon activation, the floating-gates cumulatively store the duration of the applied strain events. Defining optimal voltage thresholds plays a key role in the efficiency of the PFG sensors for structural damage identification. In this paper, symbolic dynamic analysis (SDA) based on Shannon entropy is used to find the effective voltage thresholds that ensure the maximum detectability of the structural damage-related changes. To this end, a baseline is constructed using the strain data obtained from the undamaged structure. These data are used to set the voltage threshold on every floating gate of the sensor. Then the posterior state of the structure is monitored using thresholds set upon the baseline and a cumulative density function (CDF) of strain events. In order to determine the damage severity, a damage index
\end{abstract}

Email address: Corresponding author: a.h.gandomi@stevens.edu (Amir H. Gandomi *) 
is defined based on the Euclidean norm of the distance between the CDFs for the damaged and healthy structure. The proposed technique is verified using experimental data for a steel plate subjected to an in-plane tension loading. The results confirm the capability of the proposed method in monitoring structures for damage initiation and/or propagation using the PFG sensors, and the CDFs on which the damage sensitive feature (DSF) is based can provide additional insights into the stress distributions.

Keywords: Shannon Entropy, Symbolic Dynamic Analysis, Self-powered Sensors, Wireless Damage Detection, Strain

Events, Structural Health Monitoring

2010 MSC: 00-01, 99-00

\section{Introduction}

Several different fields of science have seen advances that have culminated in methods for real time damage detection. Overall, a reliable damage detection method should have the following characteristics:

(1) it is not sensitive to environmental effects

(2) it can be applied in-situ

(3) it is economical

(4) it is able to detect and locate small damage

(5) it can be applied remotely

(6) it can monitor structures continuously

(7) it is not very sensitive to other structural features such as load variation.

The methods are of an interdisciplinary nature, and there are three main fields that must be mentioned: (1) advanced sensing technologies [1, 2]; (2) advanced damage detection algorithms $[3,4,5]$; and (3) smart materials [6]. Because of recent advances in genetic, neural network, swarm and evolutionary methodology, new damage detection algorithms have been developed. 
A new and advanced type of structural health monitoring (SHM) seeks to create smart materials which mimic biological skin by simultaneously displaying the damaged area by changing colours, as well as self-healing of the damaged region [7]. However, there are still some challenges with SHM. For instance, changes in ambient conditions can affect measured data and consequently bring about false detection. Moreover, because of high-damping characteristics of composite materials, a considerable portion of acoustic waves stemming from crack propagation would be damped. This may demand accurate placement of sensors, which means that a priori knowledge of likely damaged areas is required. Moreover, measured data from sensors are contaminated by noise and in terms of applying modal data, there is always a limitation in the number of measured modes. Although the application of smart materials for SHM is a new and promising field, it is unlikely to see real applications of these types of material in the near future.

Large Area Electronics (LAE) is another new sensing technology in the form of dense arrays that can span large areas of the structure [8]. Kong et al. propose a novel large-area strain sensing technology to monitor fatigue cracks in steel bridge structures [9]. Accordingly, the authors propose using soft elastomeric capacitors (SEC) which are flexible capacitors consisting of a dielectric layer sandwiched between to conductive layers. The so-called SEC sensor can easily be attached to the surface of the structural member. Structural strains bring about a geometric change in the sensor resulting in a corresponding change in capacitance. The authors exploit this fact to detect fatigue cracks in materials. In another study conducted by researchers at Princeton university, the authors use sensing sheets based on LAE for cyclic fatigue tests on steel plates [10].

Acoustic and Electromagnetic (EM) waves have been widely used in modern SHM techniques. Due to the characteristics of waves, there has been interest in providing an image of a defective area, or alternatively studying 
the modulation of EM waves in terms of amplitude, phase and/or frequency due to structural vibration. The key characteristic of these approaches is that they can be used for remote and continuous monitoring of structures with the aid of wave propagation and modulation within materials.

There are several methods in the literature using waves to locate damage in structures. These can be divided into two main categories based on the type of wave they deploy: methods that apply acoustic wave propagation within a material, and those that apply EM waves. The former can be divided into three branches: Acoustic Emission (AE) [11, 12, 13], Vibro-Acoustic Modulation (VAM) [14, 15, 16], and Guided waves [17, 18]. While VAM deals with the mechanical and acoustic waves interaction for damage detection, some other researchers have recently combined acoustic techniques with other methods such as eddy current for damage detection [19].

EM waves have been widely used in the context of SHM. To that end, different hardware such as antennae and radars for transmitting and receiving EM waves have been proposed [20, 21]. Self-antenna technology is a method which applies the characteristics of EM waves for damage detection. This method, which can be considered as a member of a larger category called self-sensing SHM technology, seeks to use the structure as an antenna. Accordingly, by detecting the changes in antenna characteristics an evaluation of the structural health condition is possible. For instance, CFRP structures can be modelled as half-wavelength dipole antennae. Hence, by looking at the variation of electrical or antenna properties, the structure can be monitored wirelessly at a remote location [22]. Electrical conductivity measurement has been used to monitor the health condition of structural connection points by some researchers [23].

Millimeter-wave and terahertz Doppler radar technology have been used successfully in SHM by applying radar technology and antenna theory [20, 
$21,24]$.

In a recent study, Moll et al. applied a set of transmitting and receiving antennae attached to the tower of a wind turbine to radiate EM waves towards the rotor blades. Hence, all blades can be inspected with a sensor array in a non-contact and highly automated way by exploiting the rotation of the wind turbine and the inverse synthetic aperture radar (ISAR) principle. This is a method where radar imaging is used to generate a twodimensional high resolution image of a target [20]. In order to reach to a high penetration depth in glass-fiber reinforced material, the authors applied frequency bands of $24-24.25 \mathrm{GHz}$ and $24-25.6 \mathrm{GHz}$.

Technological advances in laser technology have evolved to advance SHM during the last few decades [25]. For instance, Laser Doppler Vibrometers (LDV) have recently been used to capture non-contact vibration of bridges [26]. First the laser beam from the LDV is directed at the surface of the structure. Then, due to the Doppler shift of the reflected laser beam, the frequency and the vibration amplitude are extracted from the motion analysis of the surface. Stepped Frequency Continuous Wave (SFCW) RADAR is another method which has been used successfully to remotely extract vibration data of structures. Recently, this method has been used to detect bridge Micro-Doppler (MD) data, and to derive the natural frequency and resonance mode for a bridge while a train crosses [27].

Although, all of the methods discussed above have been applied successfully to condition monitoring of structures, problematic issues arise when it comes to applying these in real structures. For instance, AE methods need a large number of sensors to be located over a short distance on blades due to the fact that acoustic waves are rapidly damped in composite materials. In a study done by Kim et al. the pumping signal of a Vibro-Acoustic Modulation technique is provided by the blade rotation [15], but in general a source of probing signal attached to the blade is still necessary. The same 
problem arises when applying other acoustic wave methods, which makes the remote and continuous condition monitoring of structures challenging.

In this paper, a novel and effective damage detection strategy is proposed based on the application of the piezo-floating-gate (PFG) sensors, previously developed at Michigan State University [28]. The proposed method exploits a key property of the PFG sensors, namely the activation of a series of floating gates (memory cells) when the strain (or corresponding voltage) exceeds the gate preselected threshold. Accordingly, the strain-time events are cumulatively stored in the memory. Recently, some damage detection methods have been proposed based on the functionality of the PFG sensors [29]. However, that work looked generally at the mean value and variance of the strain statistics, but did not exploit the unique property of the PFG sensors that makes them highly amenable to using a Shannon entropy approach (explained further in Section 4.1). The present study on the other hand explores the feasibility of using symbolic dynamic analysis (SDA) and Shannon entropy to enhance the detectability of the structural damage-related changes from the PFG sensor data. The concept of the proposed technique can be generalised to damage detection of large structures based on the LAE concept.

The paper is organized as follows: in Section 2, the working mechanism of the PFG sensors is briefly explained; then the SDA and Shannon Entropy are discussed in Section 3 and the proposed damage detection is explained in Section 4. In Section 5, the proposed method is verified using the data from an experimental study conducted by Alavi et al. [29]. The superiority of the proposed DSF over the other probability distance measure is discussed in Section 6. Finally, Section 7 is dedicated to derived conclusions. 


\section{Self-powered piezo-floating-gate sensors}

Lead Zirconate Titanates (PZTs) are the most commonly used piezoceramics. These are solid solutions of lead zirconate and lead titanate [30]. Some specific properties can be obtained by smearing PZTs with other elements. According to the manufacturing process of these ceramics, a paraelectric to ferroelectric phase transition occurs during the cooling process. As such, piezoelectric materials can convert mechanical applied load to an electrical charge.

As far as the mechanical characteristics of PZT sensors are concerned, like ceramics, they show a high elastic modulus, brittleness and low tensile strength. The open circuit voltage $(\mathrm{V})$ generated across the PZT transducer is

$$
V=\frac{S Y D_{31} h}{\epsilon},
$$

in which $S, Y, D_{31}, h$ and $\epsilon$ represent the applied strain, Youngs modulus of the piezoelectric material, piezoelectric constant, thickness, and the electrical permittivity, respectively [31].

The piezoelectric material can be used to harvest vibration energy of structures over a wide range of frequencies. As such, piezoelectric transducers have been widely used by researchers to develop simple and efficient energy harvesting devices [32]. The piezoelectric transducers have been widely used for structural health monitoring of structures [33, 34].

The so-called PFG sensor consists of a p-channel floating-gate metaloxide-semiconductor (pMOS) transistor, which is connected to a constant current source commonly powered by the PZT transducer. Using the harvested energy on the structure due to the applied force, the electrons from the transistor channel are injected into the floating-gate, which without further energy input experiences a decay of the floating-gate voltage with respect to time. Therefore, by measuring the floating-gate voltage in longterm monitoring, one can obtain the cumulative operational time of the 
injector [35, 31].

This principle is illustrated in Figure 1. Accordingly, when a random force is applied to the structure, each of the set voltage thresholds of gates corresponding to each sensor might be exceeded from time to time (Figure 1a). However, once a bigger threshold is passed all the smaller thresholds are also passed and therefore a cumulative density function (CDF) of strain events is obtained from each sensor (Figure 1b).

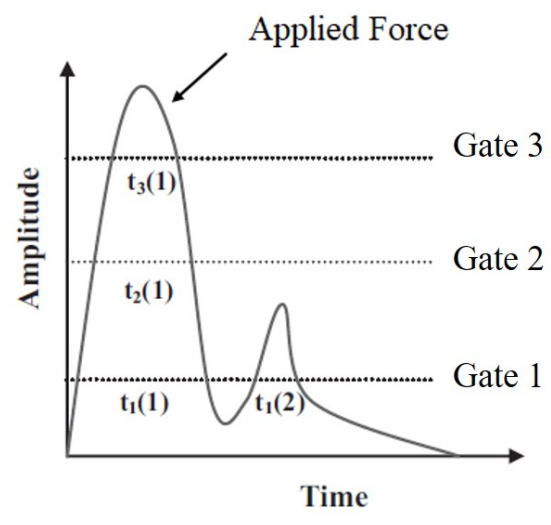

(a) Random excitation

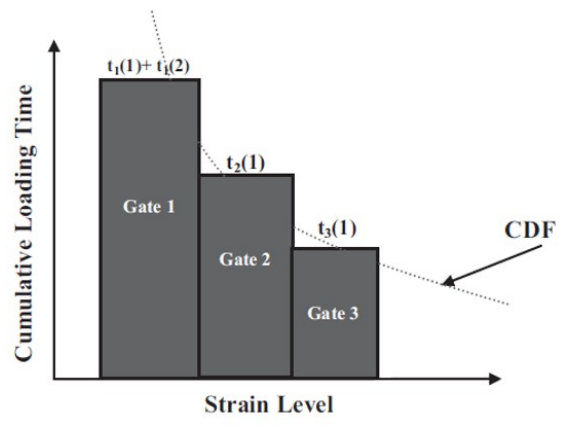

(b) cumulative time at pre-defined strain/voltage thresholds

Figure 1: A schematic representation of working procedure of PFG sensors [29].

This is due to the fact that floating-gates can store data like a nonvolatile memory and, therefore, data can be stored on board the sensor, to be retrieved remotely without a need for any external power source [36].

Further information about the function and manufacturing process of the PFG sensors can be found in [37, 35, 36, 38].

\section{Symbolic Dynamic Analysis (SDA)}

SDA, which has a root in information theory, is a one way to deal with a time series of recorded signals. In practice, for every baseline time epoch $t_{0}$ the vector of a recorded signal is sorted into some subsets representing the 
current state of the system. The dynamics of the system is studied through monitoring of the evolution of the subsets with regards to the baseline.

Researchers have applied SDA successfully to the SHM of different types of structures $[39,40]$. It has been demonstrated that SDA is superior to other methods such as Artificial Neural Networks (ANN), Principal Component Analysis (PCA), and Kernel Regression Analysis (KRA) in terms of feature extraction for damage detection [41]. SDA can also improve the signal to noise ratio considerably, which is a key factor in analysis of noisy signals $[42,43,44]$.

\subsection{Shannon Entropy}

Shannon entropy is defined as

$$
H=-\sum_{i=1}^{N} p_{i} \log _{2}\left(p_{i}\right)
$$

where $p_{i}$ is the probability of the event $i$ in the time record of all events. For example the event could be the triggering of gate $i$ in the PFG sensor.

Lemma: It can be shown that, under the general constraint of probability theorem, i.e. $\sum_{i=1}^{N} p_{i}=1$, maximum entropy is achieved when all of the incidents have equal probability. In other words, $p_{i}=p_{j}$ for any $i$ and $j$.

Proof: The above Lemma is an optimisation problem and can be proved by using the Lagrange multiplier concept. As such, a goal function can be constructed as

$$
\Lambda=-\sum_{i=1}^{N} p_{i} \log _{2}\left(p_{i}\right)-\lambda\left(\sum_{i=1}^{N} p_{i}-1\right),
$$

to be maximised without the need to explicitly parameterise the constraints. A stationary point for $\Lambda$ can be found by setting its derivatives with respect to all variables $p_{i}$ and $\lambda$ to zero where $i=1,2, \ldots, N$. 
Therefore, one can obtain

$$
\begin{aligned}
\frac{\partial \Lambda}{\partial \lambda}=0 & \Rightarrow-\sum_{i=1}^{N} p_{i}+1=0 \\
\forall i ; \frac{\partial \Lambda}{\partial p_{i}}=-\log _{2}\left(p_{i}\right)-\log _{2}(e)-\lambda=0 & \Rightarrow p_{i}=\frac{2^{-\lambda}}{e}
\end{aligned}
$$

Noting from $4 \mathrm{~b}$ that the probability $p_{i}$ is the same for all $i$, it follows from 4 a that

$$
p_{i}=\frac{1}{N} .
$$

Therefore, for a random process under the general probability condition, either a minimum or maximum Entropy is obtained when all the incidents have equal probability. It is sufficient to show by example that this is not a minimum, therefore must be a maximum. To that end, substitution of the distribution $\left\{p_{i}\right\}=\{1,0, \ldots, 0\}$ into Equation 2 gives $H=0$, which is less than the result $H=\log _{2} N$ obtained when $\left\{p_{i}\right\}=\left\{\frac{1}{N}, \frac{1}{N}, \ldots, \frac{1}{N}\right\}$ is substituted. Accordingly, for two random processes, the one that has the larger Shannon entropy is more random.

Thus, if we are to choose between two random processes to predict what the next state of the process is, we should choose the one with the larger Shannon entropy and our predictions will be less biased.

This is the key factor in symbolic time series analysis. Assume that we are interested in the evolution of a given signal through time. Accordingly, one way to deal with this problem is first to sort the signal values into some sub-signals, in which each sub-signal is a sample space. This sorting is based on thresholds chosen such that the probability of each element falling into any of these sub-signals is equal. In other words, the sub-signals contain only values within a given range, are of equal length and have a uniform distribution. This is considered as a baseline. Then, the evolution of each sub-signal in a posterior state is monitored through a similar sifting process using the same thresholds. If there has been a change of state the 
distribution will no longer be uniform. Once the distribution of data in the posterior signal is obtained a measure can be defined to obtain its distance from the initial uniform distribution.

\section{Damage detection procedure}

\subsection{The rationale behind using an Entropy-based damage indicator}

As PFG sensors only record the cumulative duration of strain events, they basically carry information about the statistical distribution of strain data in ranges defined by a set of successive strain thresholds. This perfectly matches with the concept of entropy as discussed in the section 3.1. This is the main intuition behind applying Shannon-entropy for interpreting data recorded on PFG sensors and to relate it to the health condition of the structure.

By choosing the sensor voltage (hence strain) thresholds in a way that the distribution of durations of strain events is uniform among all gates, one can continuously monitor any change in the health condition of the structure by tracing the change in the distribution of strain events. Since negligible processing of the recorded data is only required, the procedure of the damage detection will be one step ahead of other damage detection strategies.

In the following sections, the procedure of damage detection is discussed in more details.

\subsection{Setting thresholds based on Shannon entropy}

In most damage detection techniques based on the SDA, an undamaged structure is first monitored to construct a baseline for damage detection as stated in Section 3.1. After some time, the structure is monitored again to decide how much change it has undergone. The proposed PFG sensors provide the capability of real-time damage detection using SDA. To that end, it is first required that the thresholds be determined in order to be set on each sensor. 
In the stage of determining thresholds, an ordinary sensor (i.e. a piezoelectric sensor without the floating gates) must be deployed on the healthy structure at the position of interest. The sensor records the strain-time history of the structure in voltage at that position. Then the recorded data are sorted in ascending order and, according to the number of required gates, the thresholds are chosen in such a way that an equal number of data exist between any two successive thresholds. For instance, if the length of the recorded signal is $N_{s}$ and there are $N_{g}$ gates, the number of data samples in each gate is calculated approximately as $\left[\frac{N_{s}}{N_{g}}\right]$ where [.] indicates rounding to the nearest integer. Accordingly, a uniform distribution of data is obtained whose probability density function (PDF) is a flat line with probability $p(x)=\frac{1}{N_{g}}$, thus a straight line is obtained for the cumulative density function $(\mathrm{CDF})$, and one can set the smallest recorded data as the lowest threshold.

Note that the PFG sensors count the time that each threshold is passed in terms of sample count at the specified sampling frequency, and the time that is read on the $n^{\text {th }}$ gate is a cumulative time representing the sum of the times for which any larger threshold $i(i \geq n)$ is passed. Thus the CDF is actually a reverse integration of the PDF. Figure 2 shows the CDF of data that are uniformly distributed among the gates for the undamaged stage of the structure. As is evident from the graph, gates with smaller thresholds have larger corresponding value of $\mathrm{CDF}$ and vice versa.

Thus, one needs only to specify some thresholds on floating gates based on the SDA of the recorded signal obtained from the strain gauges deployed on the intact structure.

\subsection{Monitoring the structure after setting thresholds}

Once voltage thresholds for each sensor have been determined on the intact structure using ordinary piezoelectric strain gauges without the floating gates, these thresholds are set on the corresponding floating gates. These 


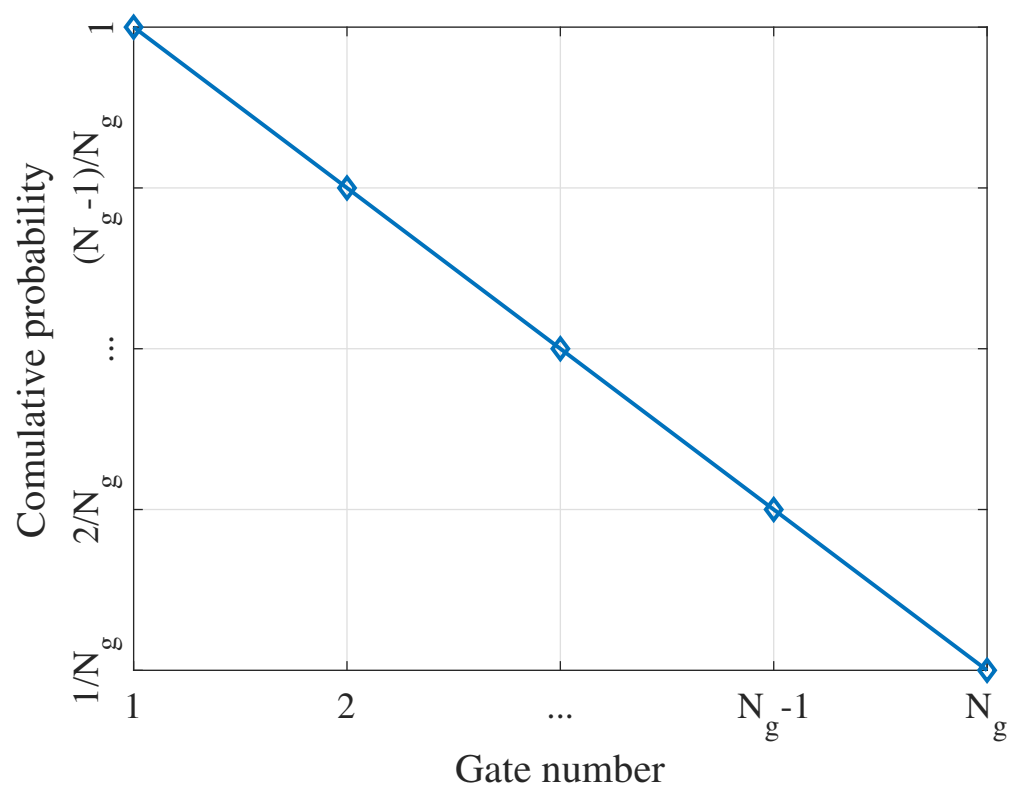

Figure 2: Diagram of stochastic characteristics of a random process versus Shannon entropy.

ordinary strain gauges are then replaced with the corresponding calibrated PFG sensor in exactly the same position.

As long as the structure is healthy, the distribution of recorded times from PFG sensors are uniform or very close to uniform. However, due to noise and uncertainty there might be always a slight deviation from a uniform distribution. As such, a Mont Carlo simulation can be performed to classify between the healthy and damaged state of the structure using some classification technique.

\subsection{Damage index}

When the posterior distribution of the data for each sensor is determined, a damage index (which we will call the damage sensitive feature, or DSF) needs to be evaluated to identify how much the structure has deviated from its healthy state. To that end, the DSF is defined in this section as the Euclidean norm of the distance between the CDF of the baseline (uniform 
distribution) and damaged states,

$$
\mathrm{DSF}=\sqrt{\sum_{i=1}^{N_{g}}\left(F_{X}^{b}\left(x_{i}\right)-F_{X}^{d}\left(x_{i}\right)\right)^{2}} .
$$

In Equation $6, F_{X}^{b}\left(x_{i}\right)$ and $F_{X}^{d}\left(x_{i}\right)$ represent the $\mathrm{CDF}$ of the strain events recorded below the $i^{\text {th }}$ threshold $x_{i}$ for the baseline and damaged structure, respectively. As is evident in Equation 6, the thresholds are the same for both healthy and damaged state of the structure, thus these are the data recorded on the PFG sensors. Figure 3, shows the flowchart of the proposed damage detection strategy adapted for the PFG sensors.

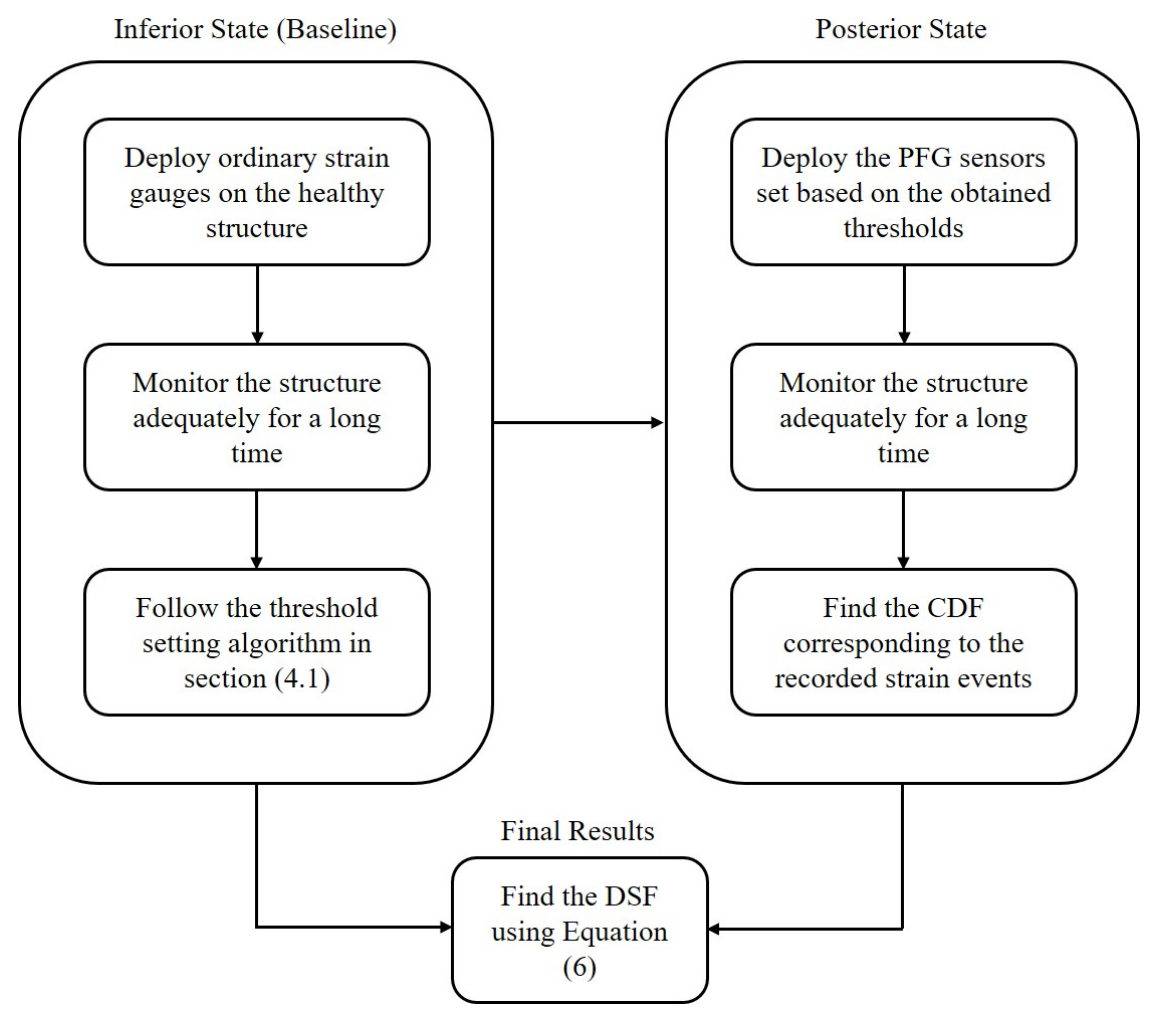

Figure 3: Flowchart of the damage detection algorithm. 


\section{Case study and results}

\subsection{Test set-up}

In this section, the proposed strategy for damage detection using the PFG sensors is tested using data obtained from a physical experiment conducted by Alavi et al. [29].

Figure 4 shows schematically the experimental set-up. As such, an A32 steel plate has been used with length 16 in (406.4 mm), clear span 12 in (304.8 $\mathrm{mm})$ between restraints, width 6 in $(152.4 \mathrm{~mm})$ and thickness 1/32 in $(0.8 \mathrm{~mm})$. As can be seen in the figure, a fixed boundary condition has been assigned to the top edge of the plate and a uniform in-plane tensile displacement was applied to the lower edge.

Four thick steel plates with dimensions 2 in $\times 6$ in $(50.8 \mathrm{~mm} \times 152.4 \mathrm{~mm})$ were installed on either side at the top and bottom of the plate to restrain the horizontal and rotational degrees of freedoms, hence to satisfy respectively the geometrical and uniform loading boundary conditions. The thick plates were attached to the specimen with three $3 / 8$ in diameter bolts at each boundary.

Crack damage was simulated by cutting a narrow horizontal slit of length $2 a$, as shown in Figure 4. Three damage scenarios are considered as shown in Table 1. The idea behind the three different slit lengths was to investigate how the measurements would change if the crack were to propagate.

\begin{tabular}{cc}
\hline Damage Scenario & Notch size ( $\mathbf{m m})$ \\
\hline$D 1$ & 17 \\
$D 2$ & 30 \\
$D 3$ & 45 \\
\hline
\end{tabular}

Table 1: Three damage scenarios are considered based on the different size of the notch $2 a$ (Figure 4). 


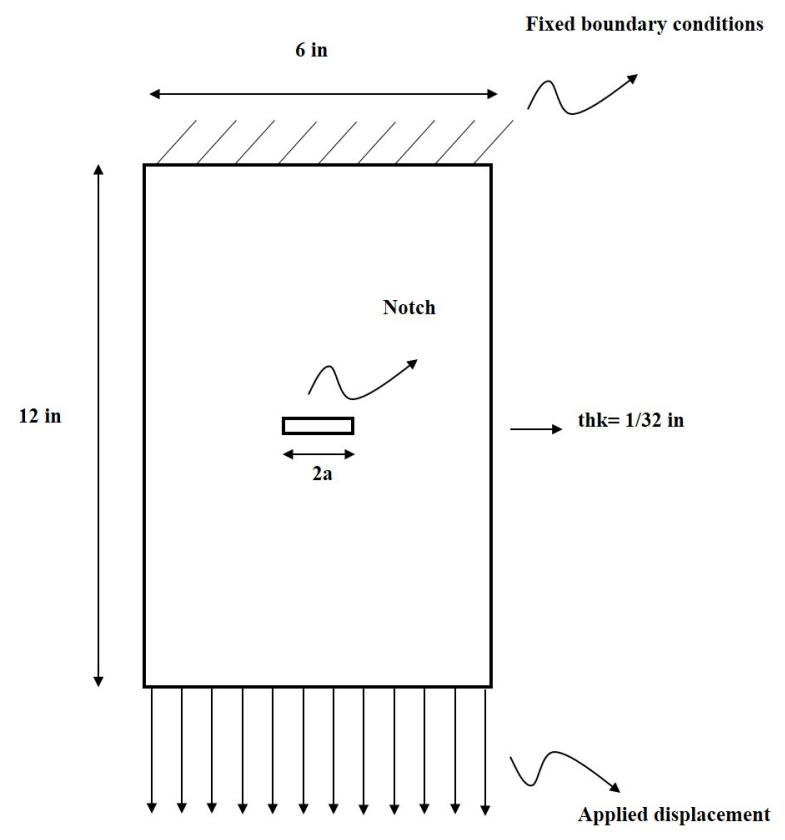

Figure 4: Experimental set-up scheme.

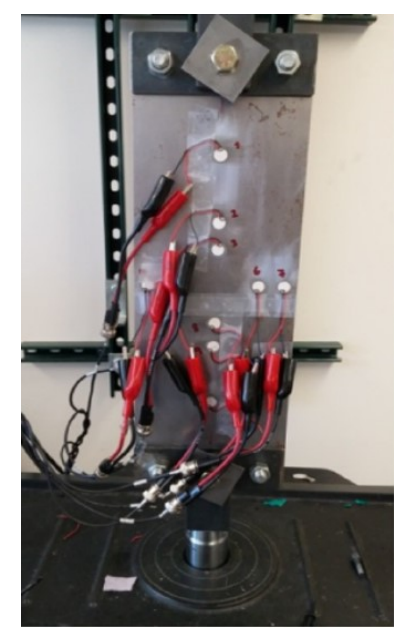

(a) Intact plate

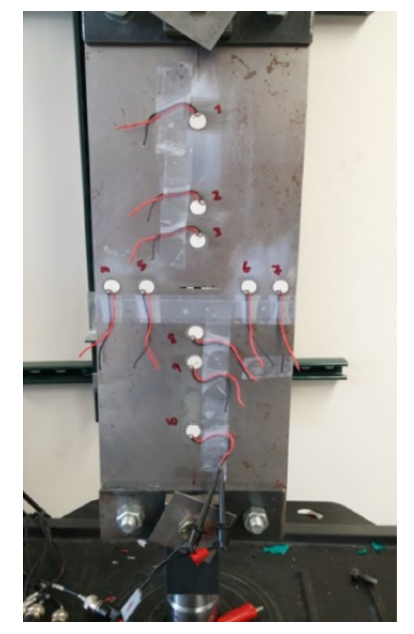

(b) Notched plate

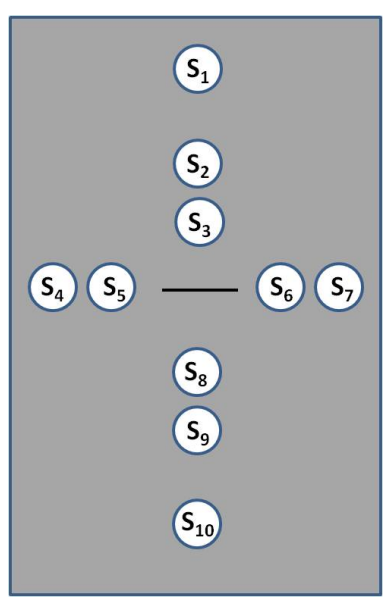

(c) Sensors arrangment

Figure 5: Sensors placement and numbering on the intact and damaged plate [29]. 
Ten strain gauges were installed on the plate to measure vertical strains along two perpendicular axes, as can be seen in Figure 5. The left figure shows conventional strain gauges attached to the intact plate, while the right figure shows the PFG sensors attached to the notched plate. Alavi et al. performed tests for the intact and three damage scenarios using both the conventional and PFG sensors, and successfully demonstrated that the two technologies gave consistent results. However, they had set the thresholds on the PFG sensors somewhat arbitrarily, so those results could not be used directly in the current work. Instead, the strain data recorded by the conventional gauges were manually sorted into the bands defined by the method outlined in Section 4.2

Alavi et al. [29] performed FE simulations using ABAQUS/CAE 6.11 in order to determine suitable location of sensors. The sensors on the vertical axis were located 1.5, 2.5 and 5.0 in $(38.1,63.5$ and $127 \mathrm{~mm})$ from the centre of the notch, and (due to the limited plate width) just 1.5 and 2.5 in on the horizontal axis.

Due to the maximum stress concentration at the crack tip, it is obvious that larger values of DSF are expected for PFG sensors 5 and 6, relative to the more remote points 4 and 7 on the same axis. On the other hand, sensors 3 and 8 would also experience significant reduction of vertical strain due to the shielding effect of the crack, thus may also be expected to show a large DSF. Sensors 2 and 9 would also show reduction but less so, and less again for 1 and 10 .

Uniaxial cyclic loading tests were performed with loading frequencies of 2 and $5 \mathrm{~Hz}$ and peak-to-peak amplitudes of 0.05 and $0.08 \mathrm{~mm}$, respectively. A preload of $4 \mathrm{kN}$ (equivalent to a displacement of $0.05 \mathrm{~mm}$ in the intact plate) ensured that the load was always tensile, however the gauges were zeroed at this preload so both positive and negative strains were measured. Only the results for $2 \mathrm{~Hz}$ frequency and $0.08 \mathrm{~mm}$ displacement amplitude 
were reported in the original paper of Alavi et al. [29], hence these are used in the current study. Further details of the experiment can be found in [29].

Seven floating-gates with different injection rates were considered for each of the 10 sensors. Hence, six meaningful data points (excluding 0 and 1) can be obtained for the cumulative probability distribution plot (Figure 2), the seventh value being used for normalisation. However, as stated above, thresholds in the original paper were not set on the sensors in the manner proposed in this study, therefore the current study manually processed data from the conventional gauges to simulate the setting of the floating gate thresholds in accordance with the procedure in Section 4.2. The results obtained from the current study are discussed in the following sections and compared with those obtained in the original work.

\subsection{Results and discussion}

Figure 6, shows the CDF of the data distribution obtained for sensors 1 to 10 .

As can be seen from the figure, and in accordance with Section 4.2 the chosen thresholds for each sensor produce a uniform distribution of data in the undamaged state. After occurring the damage, however, the distribution of the data deviates from uniform and tends to some other distribution roughly close to normal distribution. After obtaining these graphs, which can be read directly from the timers of PFG sensors, one just needs to calculate the damage sensitive feature corresponding to each state of the structure on each sensor using Equation 6 .

Note that since the PFG sensors are wireless, monitoring of the structure can be conducted in real time and therefore the damage progression can be monitored continuously.

Figure 7 shows the DSF obtained from the data read on each sensor for each of the three damage scenarios. Note that, according to the definition, 


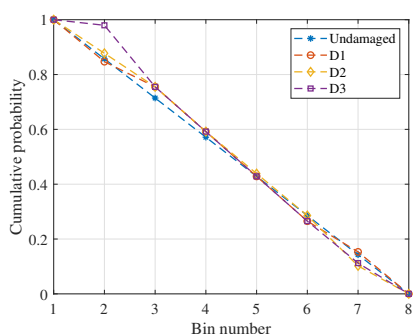

(a) $\mathrm{S} 1$

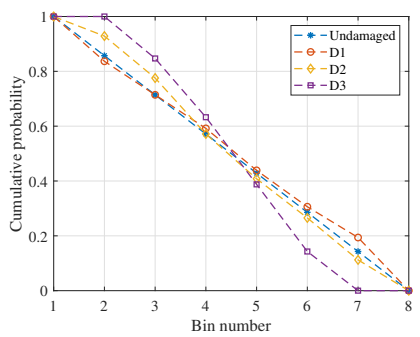

(c) S3

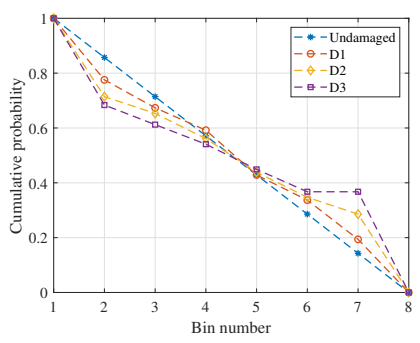

(e) S5

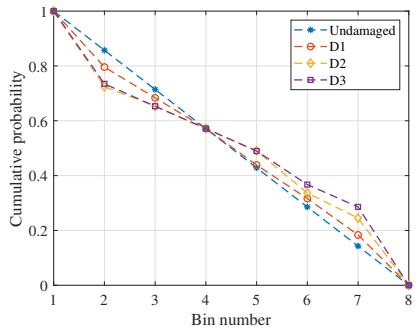

(g) S7

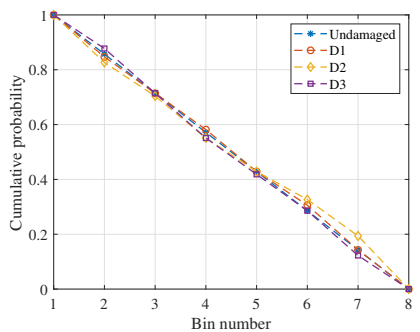

(i) S9

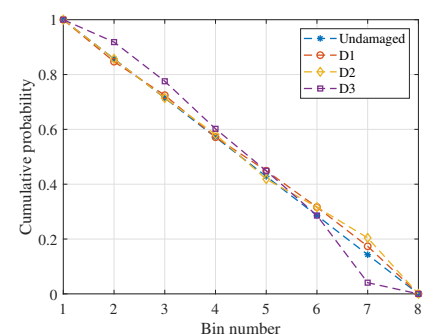

(b) $\mathrm{S} 2$

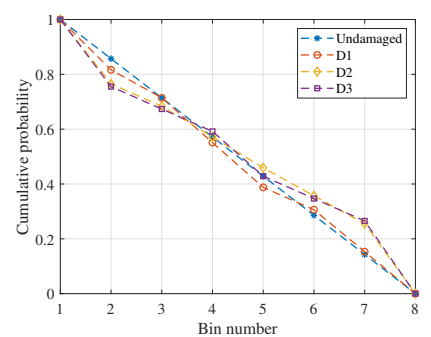

(d) S4

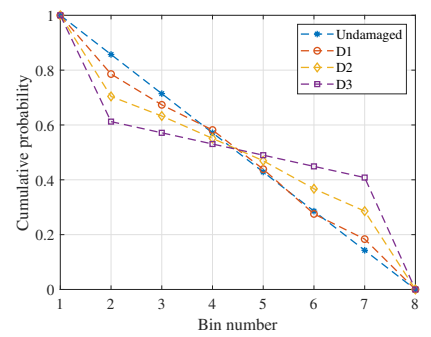

(f) S6

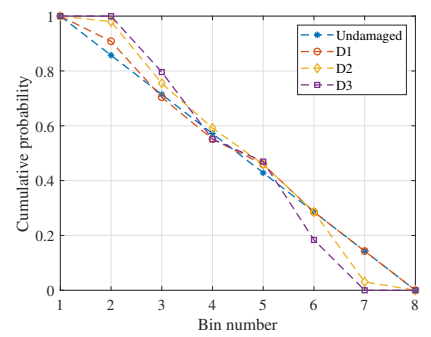

(h) S8

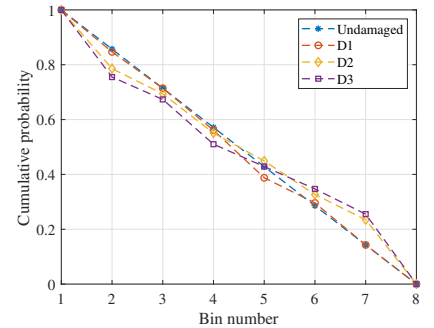

(j) S10

Figure 6: Data distribution shown by sensors. 
the DSF for the healthy state of the structure is zero and therefore is not presented in the bar-graph.

One can observe in general that sensors that are closer to the damage position show more sensitivity to the damage presence and propagation. In particular, sensors 5 and 6 , which are placed near the crack tip, show a larger DSF at each step than any of the other sensors. This is followed by sensors 3 and 8 , which are also close to the crack but are perpendicular to the damage propagation path, hence show a slightly lower DSF than sensors 5 and 6 . Thus, the DSF is greater for the case when damage is more severe, or closer to the sensor.

However, it may be observed that the calculated DSFs for sensors 9 and 10 do not seem to agree with their relative proximity to the introduced damage. This is mentioned in [29] as possibly being due to a faulty sensor. However a more likely explanation is given in Section 5.3.

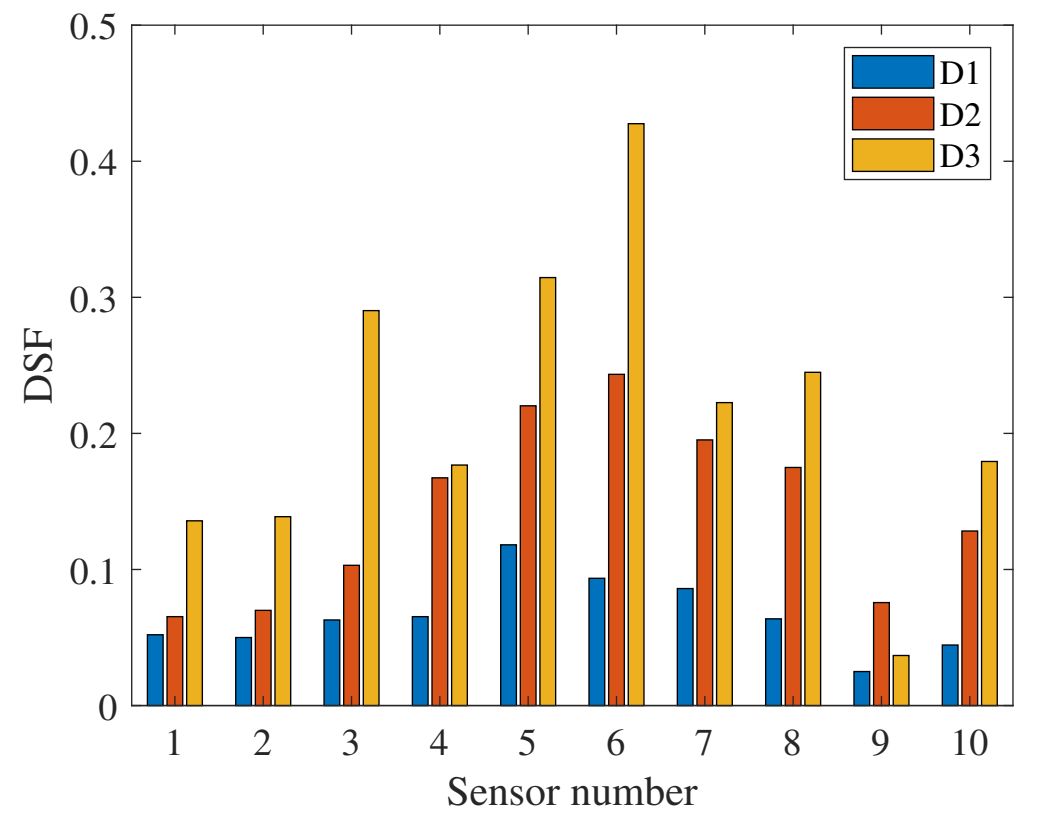

Figure 7: Calculated damage index for different sensor at different damage stages. 


\subsection{Discussion of gauges $S 9$ and $S 10$}

Sensors 3, 2 and 1 in Figure 7 show respectively a reduction of DSF with distance from the crack, as expected. With a perfect experimental setup, sensors 8, 9 and 10 should show an identical trend. However this is not borne out by the results of Figure 7, in which sensor 9 is lower than sensor 10.

To explain this, consider the corresponding cumulative probabilities shown in Figure 6.

The sigmoidal shape of gauges 1, 2 and 3 , with a positive lobe on the left and negative on the right, indicate a shift of data out of stress bins 1 and 8 and into stress bins 4 and 5 after the crack is introduced, i.e. the stresses were of lower magnitude. This is consistent with the stress shielding effect in the shadow of the crack, and the confinement of this to the crack vicinity is explained by the Saint-Venant principle.

On the other hand, gauges 4-7 show the opposite form of sigmoid, with negative lobe on the left and positive on the right, indicating shift of data into higher magnitude stress bin, i.e. a local stress concentration, as expected in the vertical stress field near the end of the crack.

Like gauge 3 , the shape of the sigmoid of gauge 8 clearly shows the expected stress shielding from the notch. However, gauge 10 (unlike its nominal counterpart, gauge 1) suggests there is a stress concentration in the vicinity of that gauge. This is likely to be a genuine effect, rather than a faulty gauge, since the neutral response of the adjacent gauge 9 is quite consistent with its location between gauges 8 and 10 .

It is speculated that the stress concentration in the vicinity of gauge 10 may be due to applied forces being concentrated at the bolts rather than being distributed uniformly across the bottom of the plate. This could arise for example if the bolts are not clamping the two restraining end plates tightly enough and there is some slippage. 


\subsection{Comparing the results with other methods}

In this section the results obtained from the current study are compared with the results obtained in the original work conducted by Alavi et al. [29].

Alavi et al. proposed the maximum voltage recorded by the PFG sensors (maximum delivered voltage, or MDV) as a damage sensitive feature. Although MDV has proven to be a good DSF to find the most critical points around the damage site, it does not seem to be a good DSF to monitor damage severity, therefore it fails to investigate the damage propagation.

For instance, in their original paper, Amir et al. showed that a greater MDV is obtained for sensors 5 and 6 and this complies well with the fact that these sensors are located at zones of maximum stress or strain concentration. However, as is evident in Figure 8, in which the obtained DSFs for each of sensors 1, 2, 3, and 8 in the current work is compared against those introduced in [29], the MDV fluctuates with damage severity and, therefore, fails to monitor the damage propagation in the material. On the other hand, as seen in Section 5.2 (and in particular Figure 7), the DSF proposed in this paper shows an increasing trend with the damage proximity, and in every case increases with the progression of its severity, which makes it a good damage indicator.

Alavi et al. also proposed a second damage detection method based on data fusion of multi-sensors. They noted that the PDF of the measured strain changed in the presence of a crack, so they first performed a Gaussian transformation to obtain the mean $(\mu)$ and standard deviation $(\sigma)$ of the distributions of the time records of each sensor output. These values changed as the damage increased, for example the stress concentration due to the crack amplified the $\sigma$ of the output signal. They then surmised that the statistics from groups of sensors might give a more reliable indication of damage. They investigated various statistical measures and found that the standard deviation of the $\mu$ and $\sigma$ values from certain groups of sensors 
(which they normalised by the highest value over the various damage scenarios, and designated 'STD of $\mu / \sigma$ of sensors') was correlated with the size of the crack. Other measures of sensor group statistics (average, minimum, maximum, range, skewness and kurtosis) were not positively correlated.

In Figures 9 and 10, the STD of $\mu$ and $\sigma$ (as presented by Alavi et al.) for the sensor groups $\{1,4,7,10\}$ and $\{1,2,3,8,9,10\}$ are compared against the mean of the DSF (calculated by Equation 6) for the same sensor groups. According to the figures the proposed damage DSF shows a much clearer correlation with the damage severity, again confirming it as a good damage indicator.

\section{Comparing the proposed DSF with other probability distance measures}

There are different methods to measure the distance between two probability distributions, which mostly measure the distance between two PDFs. In order to show why the proposed DSF, which measures the distance between two CDFs, is preferred, the following two points are considered:

1. The proposed probability distance (DSF) should be equal or close to zero for when the structure is undamaged. This means that the distance between the baseline and posterior state probability distribution functions must be zero.

2. The proposed DSF should increase as the damage becomes more severe.

In reference to the above two criteria, here we examine two different probability distances in comparison to the one proposed in this paper, which are (1) Kullback-Leibler divergence [45], and (2) Bhattacharyya distance [46]. 


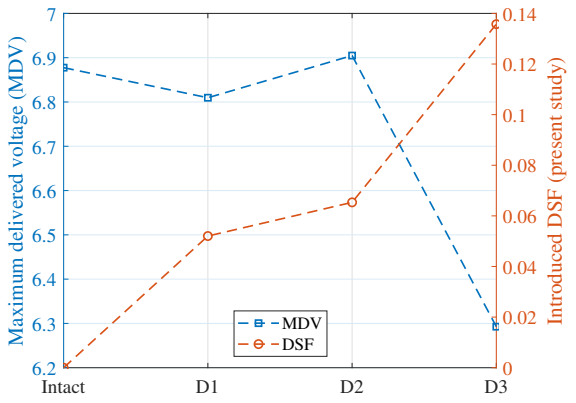

(a) S1

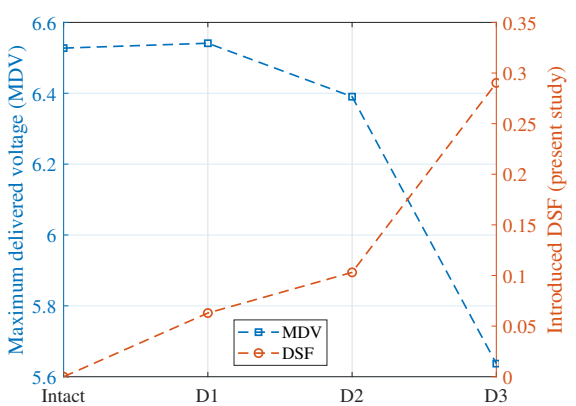

(c) $\mathrm{S} 3$

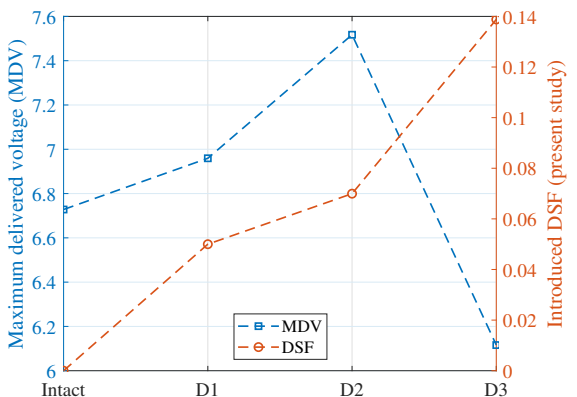

(b) S2

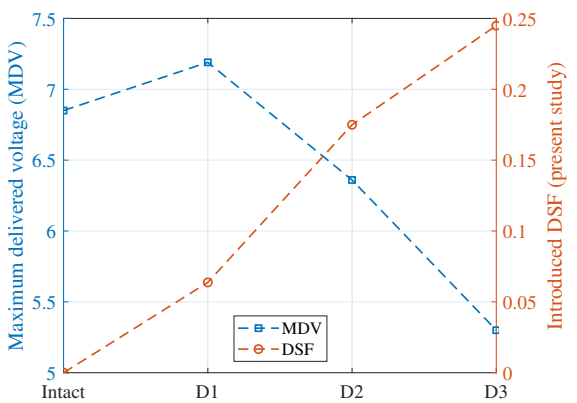

(d) $\mathrm{S} 8$

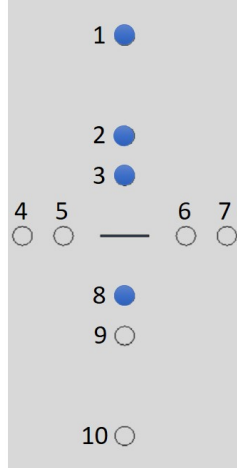

(e) locations

Figure 8: Comparing the obtained DSF for sensors 1, 2, 3, and 8 against maximum delivered voltage (MDV) obtained at this sensor in different damage scenarios. 


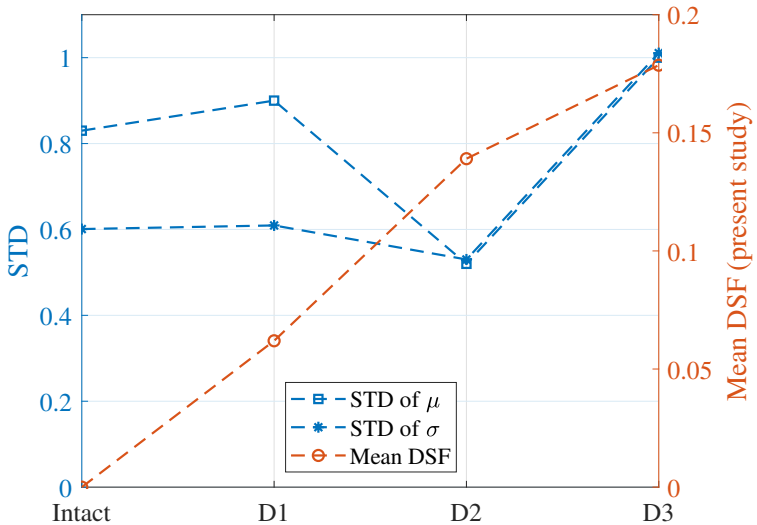

(a) STD of $\mu$ vs DSF

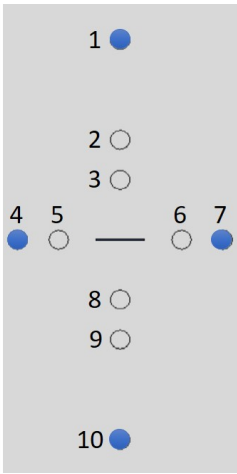

(b) locations

Figure 9: Mean value of the DSF obtained for sensors 1, 4, 7, and 10 compared to the STD of $\sigma$ and $\mu$ of the voltage obtained at these sensors.

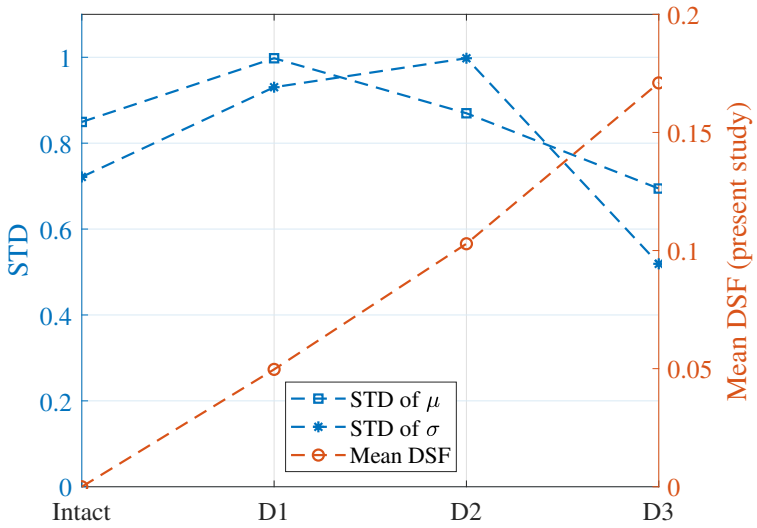

(a) STD of $\mu$ vs DSF

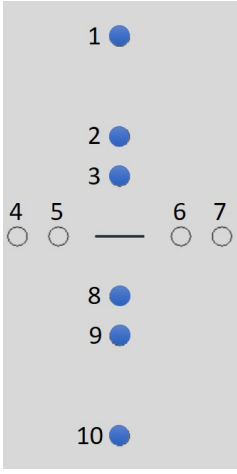

(b) locations

Figure 10: Mean value of the DSF obtained for sensors 1,2,3,8,9, and 10 compared to the STD of $\sigma$ and $\mu$ of the voltage obtained at these sensors. 


\subsection{Kullback-Leibler divergence}

The Kullback-Leibler divergence, which is also known as relative entropy and measures the difference between two probability density functions, is defined for discrete probability distributions $P$ (reference probability) and $Q$ (posterior probability) as

$$
D_{K L}(P, Q)=-\sum_{i=1}^{N} p_{i} \ln \left(\frac{p_{i}}{q_{i}}\right) .
$$

As can be seen from Equation 7, $D_{K L}$ is the expectation of the logarithmic difference between probabilities $P$ and $Q$. As such, the Kullback-Leibler divergence is defined only if for all $i$ where $q_{i}$ is zero, $p_{i}$ is also zero. In this case, the contribution of the $i^{t h}$ term vanishes as $\lim _{x \rightarrow 0} x \ln (x)=0$. However, in the problem of this paper, this is not always the case as we know that for all $i, p_{i}$ is always nonzero, whereas a zero outcome for $q_{i}$ is possible depending on the severity of damage, the number of specified gates, and/or the chosen thresholds.

Therefore, the Kullback-Leibler divergence fails to be a suitable damage sensitive feature for the current problem, even though it fulfills the first criterion.

\subsection{Bhattacharyya distance}

The Bhattacharyya distance is defined for two probability distribution functions $P$ and $Q$ over the same domain as

$$
D_{B}(P, Q)=-\ln \left(\sum_{i=1}^{N} \sqrt{p_{i} q_{i}}\right) .
$$

To show that $D_{B}$ fulfills the first criterion, we note that for the undamaged posterior state the probability distribution will be unchanged. Therefore, for all $i, q_{i}=p_{i}$, and

$$
D_{B}(P, Q)=-\ln \left(\sum_{i=1}^{N} p_{i}=1\right),
$$


which is zero since $\sum_{i=1}^{N} p_{i}=1$. Therefore, the Bhattacharyya distance appears to be a possible candidate for a damage sensitive feature.

The PDF of the posterior state of strain events was obtained from its $\mathrm{CDF}$ and the Bhattacharyya distance relative to the uniform PDF inferior state was calculated. The results are presented in the bar plot of Figure 11. It is observed,

1. The results obtained for sensor 9 complies with our discussion in Section 5.3.

2. The results obtained for sensor 1 do not comply with the damage progression from the first to second scenario.

3. Unlike the proposed damage sensitive feature based on Euclidean distance between the CDFs, the Bhattacharyya distance does not show the location of the damage properly. For instance, following our discussion at Section 5.2, it is expected that the maximum DSFs are calculated at sensors 5 and 6 (Figure 7). However, as can be seen from the Figure 11, the peak values are obtained at different sensors for different damage scenarios (for example, at 3 and 8 for D3, but at 5, 6 and 8 for D2 and at 5 and 6 for D1).

According to the above observations, we conclude that the proposed DSF based on the Euclidean distance between two CDFs outperforms the other possible methods.

\section{Conclusions}

In this paper a novel damage detection procedure is introduced for condition monitoring of structures using previously developed PFG sensors [29].

A special characteristic of these sensors has been recognized by the authors of this paper to perfectly match with the concept of Shannon Entropy. As a result, a strategy to obtain optimal values for thresholds to be set on 


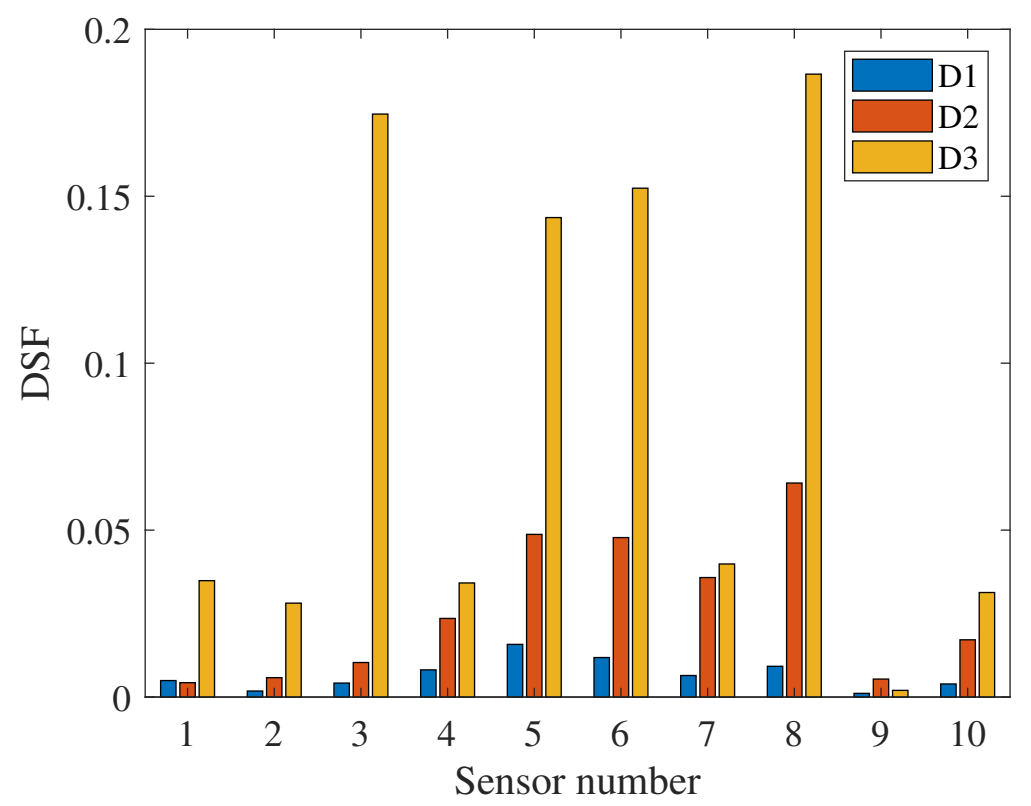

Figure 11: Calculated DSF based on the Bhattacharyya distance between the baseline and the posterior state of the strain events recorded at all sensors.

the sensors is first proposed. To that end, the baseline structure must be monitored to obtain its strain-time history. This can be done either by monitoring the real structure or studying an available FE model of the structure. As such, the structure needs to be monitored for strain at the position of interest for some time while it is operating in its natural operation condition, to ensure that all loading scenarios are considered.

After obtaining the thresholds, they need to be set on the gates for each sensors. Then, a DSF has been introduced to find the location and severity of damage. The proposed damage detection procedure is shown to be perfectly matched with the special characteristic of the PFG sensors.

In this paper, results obtained from a uniaxial cyclic loading test on a damaged plate were used to demonstrate the ability of the proposed method. However, the proposed method can also be applied to real structure subjected to random ambient vibration, due to the fact that any random white noise is a linear superposition of infinite harmonics. This also complies bet- 
ter with the nature of SDA and Shannon Entropy, which work ideally when dealing with a random process. It should also be mentioned that any state of the structure can be considered as a baseline, and therefore one can monitor any possible damage propagation at the vicinity of the sensor position.

It has been shown that the proposed DSF is sensitive enough to determine both the damage location and severity. The results obtained from this work were compared to results presented in [29]. Accordingly, it has been demonstrated that the proposed method is more effective. Furthermore, the CDFs on which the DSF is based can provide additional insights into the stress distributions, such as those discussed in Section 5.3.

Finally, it has been discussed why the proposed probability distance measure between two CDFs is a good DSF. It has been demonstrated that the use of Euclidean distance for the proposed DSF outweighs some other well known probability distance measures which could have been used.

The proposed damage detection strategy along with the exploited sensing technology has been proven to work well for structural health monitoring of structures. As such, not only can the proposed strategy be used for damage detection on new structures, but it can be also used for monitoring damage in old structures. This can be achieved by considering the current state of the structure to be the primary state and basis for the choice the voltage thresholds. As damage is a dynamic phenomenon and progresses through time any change in the calculated DSF can be referred to presence of damage at the vicinity of sensors.

There are a few questions, however, which need to be addressed in future works. For instance, one can study the possibility of correlating the value of the proposed DSF to the possible location of the damage. Further future work could focus on developing a strategy to find the exact location of damage by determining the intersection of likely circular damage zones of specific radius obtained from the different sensors. A new electronic device 
can be also developed based on the proposed damage detection strategy to show the severity of damage recorded on each sensor. This can be shown either on a display monitor attached to sensors or transferred wirelessly to a remote monitoring unit.

\section{Conflict of interest}

The authors certify that there is no conflict of interest.

\section{References}

[1] B. Glišić, Y. Yao, S.-T. E. Tung, S. Wagner, J. C. Sturm, N. Verma, Strain sensing sheets for structural health monitoring based on largearea electronics and integrated circuits, Proceedings of the IEEE 104 (8) (2016) 1513-1528.

[2] A. Downey, S. Laflamme, F. Ubertini, H. Sauder, P. Sarkar, Experimental study of thin film sensor networks for wind turbine blade damage detection, AIP Conference Proceedings 1806 (1) (2017) 070002. doi:10.1063/1.4974617.

[3] Z. Dzunic, J. G. Chen, H. Mobahi, O. Buyukozturk, J. W. Fisher III, A Bayesian state-space approach for damage detection and classification, in: Dynamics of Civil Structures, Volume 2, Springer, 2015, pp. 171183.

[4] H. Turnbull, P. Omenzetter, Damage severity assessment in wind turbine blade laboratory model through fuzzy finite element model updating, in: SPIE Smart Structures and Materials+ Nondestructive Evaluation and Health Monitoring, International Society for Optics and Photonics, 2017, pp. 101692E-101692E. 
[5] S. Hoell, P. Omenzetter, Optimal selection of autoregressive model coefficients for early damage detectability with an application to wind turbine blades, Mechanical Systems and Signal Processing 70 (2016) $557-577$.

[6] E. Haentzsche, R. Mueller, M. Huebner, T. Ruder, R. Unger, A. Nocke, C. Cherif, Manufacturing technology of integrated textile-based sensor networks for in situ monitoring applications of composite wind turbine blades, Smart Materials and Structures 25 (10) (2016) 105012.

[7] A. S. Ahmed, R. V. Ramanujan, Magnetic field triggered multicycle damage sensing and self healing, Scientific reports 5. doi:https:// doi.org/10.1038/srep13773.

[8] S. Shang, G. J. Yun, P. Qiao, Delamination identification of laminated composite plates using a continuum damage mechanics model and subset selection technique, Smart Materials and Structures 19 (5) (2010) 055024 .

[9] X. Kong, J. Li, W. Collins, C. Bennett, S. Laflamme, H. Jo, A largearea strain sensing technology for monitoring fatigue cracks in steel bridges, Smart Materials and Structures 26 (8) (2017) 085024.

[10] V. Kumar, L. E. Aygun, N. Verma, J. C. Sturm, B. Glisic, Sensing sheets based on large area electronics for structural health monitoring of bridges, in: Sensors and Smart Structures Technologies for Civil, Mechanical, and Aerospace Systems 2019, Vol. 10970, International Society for Optics and Photonics, 2019, p. 109702G.

[11] J. Tang, S. Soua, C. Mares, T.-H. Gan, An experimental study of acoustic emission methodology for in service condition monitoring of wind turbine blades, Renewable Energy 99 (2016) 170-179. 
[12] Z. Bo, Z. Yanan, C. Changzheng, Acoustic emission detection of fatigue cracks in wind turbine blades based on blind deconvolution separation, Fatigue \& Fracture of Engineering Materials \& Structures 40 (6) (2017) 959-970.

[13] N. K. Tsopelas, D. G. Papasalouros, A. A. Anastasopoulos, D. A. Kourousis, J. W. Dong, Acoustic emission for structural integrity assessment of wind turbine blades, in: Advances in Acoustic Emission Technology, Springer, 2015, pp. 369-382.

[14] L. Pieczonka, A. Klepka, A. Martowicz, W. J. Staszewski, Nonlinear vibroacoustic wave modulations for structural damage detection: an overview, Optical Engineering 55 (1) (2016) 011005-011005.

[15] S. Kim, D. E. Adams, H. Sohn, G. Rodriguez-Rivera, N. Myrent, R. Bond, J. Vitek, S. Carr, A. Grama, J. J. Meyer, Crack detection technique for operating wind turbine blades using vibro-acoustic modulation, Structural Health Monitoring 13 (6) (2014) 660-670.

[16] T. Ooijevaar, M. D. Rogge, R. Loendersloot, L. Warnet, R. Akkerman, T. Tinga, Vibro-acoustic modulation-based damage identification in a composite skin-stiffener structure, Structural Health Monitoring 15 (4) (2016) 458-472.

[17] M. Mitra, S. Gopalakrishnan, Guided wave based structural health monitoring: A review, Smart Materials and Structures 25 (5) (2016) 053001.

[18] Z. Su, C. Yang, N. Pan, L. Ye, L.-M. Zhou, Assessment of delamination in composite beams using shear horizontal ( $\mathrm{SH}$ ) wave mode, Composites Science and Technology 67 (2) (2007) 244-251.

[19] V. Mishakin, V. Klyushnikov, A. Gonchar, M. Kachanov, On assessing damage in austenitic steel based on combination of the acoustic and 
eddy current monitoring, International Journal of Engineering Science 135 (2019) 17-22.

[20] J. Moll, P. Arnold, M. Mälzer, V. Krozer, D. Pozdniakov, R. Salman, S. Rediske, M. Scholz, H. Friedmann, A. Nuber, Radar-based structural health monitoring of wind turbine blades: The case of damage detection, Structural Health Monitoring 17 (4) (2018) 815-822.

[21] M. Scholz, S. Rediske, A. Nuber, H. Friedmann, J. Moll, P. Arnold, V. Krozer, P. Kraemer, R. Salman, D. Pozdniakov, Structural health monitoring of wind turbine blades using radar technology: First experiments from a laboratory study, in: 8th European Workshop On Structural Health Monitoring (EWSHM 2016), 2016, pp. 1-10.

[22] R. Matsuzaki, A. Todoroki, Wireless damage detection of CFRP using self-antenna technology, Proceedings of ICCM 2009.

[23] I. Argatov, I. Sevostianov, Health monitoring of bolted joints via electrical conductivity measurements, International Journal of Engineering Science 48 (10) (2010) 874-887.

[24] J. Moll, K. Bechtel, B. Hils, V. Krozer, Mechanical vibration sensing for structural health monitoring using a millimeter-wave Doppler radar sensor, in: EWSHM-7th European Workshop on Structural Health Monitoring, 2014, pp. 1802-1808.

[25] S. Rothberg, M. Allen, P. Castellini, D. Di Maio, J. Dirckx, D. Ewins, B. J. Halkon, P. Muyshondt, N. Paone, T. Ryan, et al., An international review of laser Doppler vibrometry: Making light work of vibration measurement, Optics and Lasers in Engineering 99 (2017) 11-22.

[26] E. K. Ervin, V. Aranchuk, J. Chambers, et al., Final project report: Rapid non-contact measurement using multiple point laser doppler vi- 
brometry for health evaluation of rail and road bridges, Tech. rep., University of Mississippi (2015).

[27] H. Huang, P. Xu, Bridge micro doppler phenomenon analysis by using sfcw radar system, in: Microwave and Millimeter Wave Technology (ICMMT), 2016 IEEE International Conference on, Vol. 2, IEEE, 2016, pp. $874-876$.

[28] S. Chakrabartty, N. Lajnef, N. Elvin, A. Gore, Self-powered sensor, US Patent 8,056,420 B2 (Nov. 15 2011).

URL https://patents.google.com/patent/US8056420B2

[29] A. H. Alavi, H. Hasni, N. Lajnef, K. Chatti, Damage growth detection in steel plates: Numerical and experimental studies, Engineering Structures 128 (2016) 124-138.

[30] J. Sirohi, I. Chopra, Fundamental understanding of piezoelectric strain sensors, Journal of Intelligent Material Systems and Structures 11 (4) (2000) 246-257.

[31] N. Lajnef, M. Rhimi, K. Chatti, L. Mhamdi, F. Faridazar, Toward an integrated smart sensing system and data interpretation techniques for pavement fatigue monitoring, Computer-Aided Civil and Infrastructure Engineering 26 (7) (2011) 513-523.

[32] A. Abdelkefi, Aeroelastic energy harvesting: A review, International Journal of Engineering Science 100 (2016) 112-135.

[33] K. K. Tseng, L. Wang, Structural damage identification for thin plates using smart piezoelectric transducers, Computer methods in applied mechanics and engineering 194 (27-29) (2005) 3192-3209.

[34] I. Benedetti, M. Aliabadi, A. Milazzo, A fast BEM for the analysis of damaged structures with bonded piezoelectric sensors, Computer 
Methods in Applied Mechanics and Engineering 199 (9-12) (2010) 490501.

[35] C. Huang, N. Lajnef, S. Chakrabartty, Calibration and characterization of self-powered floating-gate usage monitor with single electron per second operational limit, IEEE Transactions on Circuits and Systems I: Regular Papers 57 (3) (2010) 556-567.

[36] W. Borchani, K. Aono, N. Lajnef, S. Chakrabartty, Monitoring of postoperative bone healing using smart trauma-fixation device with integrated self-powered piezo-floating-gate sensors, IEEE Transactions on Biomedical Engineering 63 (7) (2016) 1463-1472.

[37] N. Lajnef, K. Chatti, S. Chakrabartty, M. Rhimi, P. Sarkar, et al., Smart pavement monitoring system, Tech. rep., United States Federal Highway Administration (2013).

[38] S. Chakrabartty, T. Feng, K. Aono, Gen-2 RFID compatible, zero down-time, programmable mechanical strain-monitors and mechanical impact detectors, in: Sensors and Smart Structures Technologies for Civil, Mechanical, and Aerospace Systems 2013, Vol. 8692, International Society for Optics and Photonics, 2013, p. 86921A.

[39] M. Makki Alamdari, B. Samali, J. Li, Y. Lu, S. Mustapha, Structural condition assessment using entropy-based time series analysis, Journal of Intelligent Material Systems and Structures (2017) 1045389X16679288.

[40] J. G. Brida, L. F. Punzo, Symbolic time series analysis and dynamic regimes, Structural Change and Economic Dynamics 14 (2) (2003) 159 183.

[41] C. Rao, A. Ray, S. Sarkar, M. Yasar, Review and comparative evalu- 
ation of symbolic dynamic filtering for detection of anomaly patterns, Signal, Image and Video Processing 3 (2) (2009) 101-114.

[42] P. Beim Graben, Estimating and improving the signal-to-noise ratio of time series by symbolic dynamics, Physical Review E 64 (5) (2001) 051104 .

[43] C. S. Daw, C. E. A. Finney, E. R. Tracy, A review of symbolic analysis of experimental data, Review of Scientific instruments 74 (2) (2003) 915-930.

[44] V. Rajagopalan, A. Ray, R. Samsi, J. Mayer, Pattern identification in dynamical systems via symbolic time series analysis, Pattern Recognition 40 (11) (2007) 2897-2907.

[45] S. Kullback, R. A. Leibler, On information and sufficiency, The Annals of Mathematical Statistics 22 (1) (1951) 79-86.

[46] A. Bhattacharyya, On a measure of divergence between two statistical populations defined by their probability distributions, Bull. Calcutta Math. Soc. 35 (1943) 99-109. 